\title{
Event-based Motion Coordination of Multiple Underwater Vehicles Under Disturbances
}

\author{
Pedro V. Teixeira, Dimos V. Dimarogonas, Karl H. Johansson and João Sousa
}

\begin{abstract}
The problem of driving a formation from an initial to a target configuration while under the effect of external disturbances is studied. Additional restrictions on agent sensing as well as inter-agent communication must be satisfied. We present a leader-follower solution that relies on a simple uncertainty model to trigger surfacing events. These events are then used to update the control signal, for which two different, provably correct, control strategies are proposed. Finally, we show how the surfacing events can be used to characterize the disturbance set. Numerical examples on relevant scenarios are also provided.
\end{abstract}

\section{INTRODUCTION}

Recent technological developments have allowed for the appearance of low-cost robotic platforms. Motivated by the advantages of multi-agent systems, the spectrum of multiagent applications has been increasing, sparking the interest of the research community and leading to greater advances in this field. A particular example is the increased data redundancy and the reduced time and cost needed to cover a wider area that come with the use of several low-cost platforms compared to the use of a single (often expensive) platform.

However, with the use of a vehicle formation inevitably comes the problem of agent coordination, which becomes more complex if the platforms used have limited communication and sensing capabilities. This is usually the case for low-cost vehicles - underwater communication is constrained in terms of range and bandwidth, with underwater acoustic modems requiring considerable amounts of power. At the same time, underwater positioning poses another challenge as good inertial sensors are very expensive and acoustic positioning systems have a limited range. Due to these limitations, most low-cost platforms have to surface to get position fixes through the use of GPS. Communication is also limited to these intervals, as more efficient, low-power wireless modules can be used. Such an example is the Underwater Systems and Technologies Laboratory (USTL - http://whale.fe.up.pt/lsts/) fleet, which includes six low-cost Light Autonomous Underwater Vehicles (LAUVs), equipped with a simple inertial navigation system, GPS, and long baseline (LBL) system by default.

Pedro V. Teixeira and João Sousa are with the Underwater Systems and Technologies Laboratory, Faculty of Engineering of the University of Porto, Porto, Portugal (pvazteixeiradieee.org, jtasso@fe.up.pt). Dimos V. Dimarogonas is with the Laboratory for Information and Decision Systems,Massachusetts Institute of Technology, Cambridge, MA, U.S.A. (ddimar@mit.edu). Karl H. Johansson is with the KTH ACCESS Linnaeus Center, School of Electrical Engineering, Royal Institute of Technology (KTH), Stockholm, Sweden (kallejdee.kth.se). This work was supported in part by the European Project FeedNetBack, the Swedish Research Council, the Swedish Strategic Research Foundation and the Portuguese Foundation for Science and Technology NETV project.
Additionally, they can be configured with CTD, side-scan sonar and underwater acoustic modems. Although the LAUV is undergoing development, it has been successfully deployed several times over the last few years.

Motivated by the above observations, the problem of multiagent coordination for this particular type of vehicles can be summarized as that of finding a control strategy that is able to drive a formation of AUVs from the initial to the target set of positions within a given time and while under the effect of external disturbances such as ocean currents. At the same time, inter-agent communication and position feedback have to be limited to the instants where the agents have surfaced.

Several formation control designs have been proposed. Some examples can be found in [1]-[6]. For underwater vehicle applications, in particular, solutions to the multi-agent problem based on the use of artificial potentials and virtual bodies are presented in [7], [8]. Lyapunov methods are also used, particularly for path-following purposes [9]. In [10] the problem is studied from a high-level perspective, and a hierarchical control architecture is proposed for the centralized control of a team of vehicles.

In this note we propose some extensions with respect to our previous work in [11], namely, we let the upper bound on the uncertainty growth rate be time-varying, rendering our approach more robust to unknown or time varying disturbance sets. Then, we present a method to estimate the disturbance set, based on the several position drift measurements available at each surfacing instant.

In this article we begin by studying the single-agent problem, where we use a position uncertainty model to develop an event-triggered control strategy based on a boundedness assumption on the disturbance set. In contrast to our previous work, we let this bound be time varying. This boundedness assumption allows us to determine when (and where) the agent should surface and get a position fix in order to prevent its position uncertainty from exceeding a threshold value. As feedback is also limited to these instants, the control signal will only be updated at the surfacing events. Two different control laws are proposed for this scenario, and are then extended and proven to work for a multi-agent formation. Finally, we take advantage of the surfacing events to characterize the disturbances acting on the vehicle.

This note is organized as follows: in Section II we present the agent model, information structure and some background concepts, all of which are used to present the problem definition. Our proposed event-triggered solution to the simplified, single-agent version of the original problem is briefly revisited in Section III. Here, in contrast to our previous work [11], 
we use a more robust mechanism to trigger the update of the control signal. Two provably correct control strategies and corresponding sufficient conditions for target reachability are also derived. This approach is then extended to the original formation problem in Section IV, and sufficient conditions under which such extension is valid are derived. In Section $\mathrm{V}$ we discuss how to characterize the disturbance set using the agents' position drifts, followed by the presentation and discussion of some relevant numerical examples in Section VI.

\section{Problem Statement}

In order to maintain the self-containment of the current note, we revisit the problem formulation of [11]. Our formation consists of a set of $M$ planar $\left(x^{j} \in \mathbb{R}^{2}\right)$ agents, $A=\left\{a^{1}, a^{2}, \ldots, a^{M}\right\}$, that are located at the initial set $X_{0}=\left\{x^{1}\left(t_{0}\right), x^{2}\left(t_{0}\right), \ldots, x^{j}\left(t_{0}\right), \ldots, x^{N}\left(t_{0}\right)\right\}$ at time $t_{0}$. After applying a transformation to the unicycle model [12], and assuming additive disturbances, each agent $a^{j}$ can be modeled as a perturbed single integrator:

$$
\dot{x}^{j}(t)=u^{j}(t)+\omega^{j}(t),
$$

with $u(t) \in \mathcal{U}(t)$ (the admissible control set) and $\omega(t) \in$ $\Omega(t)$ (the disturbance set), for $t \in\left[t_{0}, t_{T}\right]$. The use of such a simple model is justified the fact that we are interested in path planning and not attitude control. A possible definition for the admissible control set comes from the AUV's maximum linear speed, $u_{\max }$ :

$$
\mathcal{U}=\left\{u \in \mathbb{R}^{2}:\|u\| \leq u_{\max }\right\}
$$

The disturbance set will, for now, be left undefined.

As has already been mentioned, we assume the agents have very limited sensing capabilities - each agent can only measure its own position when stopped (at the surface). Communication between agents is also limited to these instants, and can only take place if the two agents are connected. For this purpose, we let the network graph be described using a simple communication model, where the network's adjacency matrix $A$ at time $t$ is defined by

$$
a_{j, k}(t)=\left\{\begin{array}{l}
1, \text { if }\left\|x^{j}(t)-x^{k}(t)\right\| \leq r \\
0, \text { otherwise } .
\end{array}\right.
$$

where $r$ denotes the communication range, assumed to be the same for all agents.

We can now formally state our problem as that of finding $u^{j}(t)$ for a set of $M$ agents, $A=\left\{a^{1}, a^{2}, \ldots, a^{j}, \ldots, a^{M}\right\}$ that drives the formation from the initial configuration $X_{0}=$ $\left\{x^{1}\left(t_{0}\right), x^{2}\left(t_{0}\right), \ldots, x^{j}\left(t_{0}\right), \ldots, x^{M}\left(t_{0}\right)\right\}$ to the desired configuration $X_{T}=\left\{x_{T}^{1}, x_{T}^{2}, \ldots, x_{T}^{j}, \ldots, x_{T}^{M}\right\}$, within the desired time, $t_{T}$, and while satisfying the above conditions on communication and measurement.

\section{The Single Agent Case}

We begin by revisiting the equivalent single agent problem, and present the framework first developed in [11] in order to keep this paper self-contained. We will, however, modify it so that it can deal with time-varying bounds.

We want to find a control strategy to drive an agent $a$ from an initial position $x\left(t_{0}\right)$ to a target position $x_{T}$ within the specified time, $t_{T}$. Note that here too position measurement is restricted to the intervals where the agent is stopped. Since feedback is limited to these instants, it is natural to assume that the control signal will be a piecewise constant control signal:

$$
u(t)=\bigcup_{i=0}^{N-1} u_{i}
$$

where $u_{i}:\left[t_{i}, t_{i+1}\right] \rightarrow \mathbb{R}^{2}, \forall i \in\{0,1, \ldots, N-1\}$. Under this assumption, the solution to equation (1) can be written as

$$
x\left(t_{N}\right)=x_{0}+\sum_{i=0}^{N-1} u_{i}\left(t_{i+1}-t_{i}\right)+\sum_{i=1}^{N} \delta_{i}
$$

where $\delta_{i+1}$ is the position drift from $t_{i}$ to $t_{i+1}$ :

$$
\delta_{i+1}=\int_{t_{i}}^{t_{i+1}} \omega(t) d t
$$

In what follows we will make two assumptions (we will deal with how to fulfill them later on): $i$ ) we have available a control law $h(\cdot)$ that is able to drive the agent from $x_{0}$ to $x_{T}$ if there are no disturbances; and $\left.i i\right)$ at every instant $t_{i}$, the agent has access to an upper bound on the disturbance speed:

$$
\gamma_{i} \geq \max _{\omega \in \Omega, t \in\left[t_{i}, t_{i+1}\right]}(\|\omega(t)\|)
$$

$\gamma_{i}$ can also be interpreted as an upper bound on the position uncertainty growth rate. Due to this fact, the minimum time it will take for the agent's position uncertainty to reach a threshold value $\epsilon$ will be given by

$$
\Delta t_{i}=\epsilon \gamma_{i}^{-1}
$$

We call $\epsilon$ the maximum position uncertainty. As we do not want position uncertainty to exceed this value, it is reasonable to have the agent surface to get a position fix after traveling for $\Delta t_{i}$ time units since the last fix ( $\Delta t_{i}$ is called the $i^{\text {th }}$ travel time). As such, the $i^{t h}$ surfacing instant will be:

$$
t_{i}=t_{i-1}+\Delta t_{i-1}
$$

or, alternatively,

$$
t_{i}=t_{0}+\sum_{k=0}^{i-1} \Delta t_{k}
$$

At $t_{i}$ the agent will have access to $x_{i}$, and will use it compute $u_{i}$ through the $h(\cdot)$ control law. Ideally, this would cause the agent to surface at a point

$$
W_{i+1}=x_{i}+u_{i} \Delta t_{i}
$$

that is, the $i+1^{\text {th }}$ waypoint. Being that there are disturbances, the agent will surface at

$$
x_{i+1}=W_{i+1}+\delta_{i+1}
$$


However, and because of our definition of $\Delta t_{i}$ (equation (8)) we can guarantee that

$$
\left\|\delta_{i}\right\| \leq \epsilon, \forall i \in\{0,1, \ldots, N\}
$$

Using this construction, there will be an instant for which the time the uncertainty takes to reach the threshold value will be greater than or equal to the time left to reach the target. As such, we let $N-1$ be such that $\gamma_{N-1}^{-1} \epsilon \geq t_{T}-t_{N-1}$, and define $\Delta t_{N-1}=\min \left(t_{T}-t_{N-1}, \gamma_{N-1}^{-1} \epsilon\right)$. Consequently, we have that $t_{N}=t_{N-1}+\Delta t_{N-1}=t_{T}$.

Having devised an uncertainty model that allows us to determine when to surface and get a position fix so that we can update the control signal, we now turn to the control law itself.

In the unperturbed scenario - i.e., where $\omega(t)=0, t \in$ $\left[t_{0}, t_{T}\right]$ - setting

$$
u_{0}=\left(x_{T}-x_{0}\right)\left(t_{T}-t_{0}\right)^{-1}
$$

would yield the optimal control signal, that is, the signal with the smallest $\|u\|$ that is able to successfully drive the agent to the target in the specified time. With this in mind, we could apply this strategy at every surfacing instant $t_{i}$ :

$$
u_{i}=\left(x_{T}-x_{i}\right)\left(t_{T}-t_{i}\right)^{-1}, \forall i \in\{0,1, \ldots, N-1\}
$$

We call this control strategy $h_{1}$ and the main results for it follow.

Theorem 1 (The $h_{1}$ control strategy): Consider the system described by (1) and controlled by (15), with $t_{i}$ defined as in (9), Under these assumptions, the following properties hold: $i$ ) $W_{N}=x_{T}$; and $\left.i i\right)\left\|x_{i}-W_{i}\right\| \leq \epsilon, i \in\{1, \ldots, N\}$. Assuming $\mathcal{U}$ defined as in (2), a sufficient condition for this theorem to hold is

$$
\left\|u_{0}\right\|\left(1+\sum_{k=0}^{N-1} \frac{\epsilon}{\left\|u_{0}\right\|}\left(t_{T}-t_{k}\right)^{-1}\right) \leq u_{\max }
$$

Proof: The proof is omitted as it is a simple extension of the proof of theorem 1 in [11].

Looking at theorem 1's condition, we can see that a an adverse disturbance will cause $\left\|u_{i}\right\|$ to increase monotonically. This is caused by the fact that the proposed control strategy takes too long to compensate for a given position drift, which, in the mentioned adverse scenarios will cause the control signal to increase. If we compensate for $\delta_{i}$ during $\Delta t_{i}$ (as opposed to $\left.\left[t_{i}, t_{T}\right]\right)$, that is

$$
u_{i}=u_{0}-\delta_{i} \Delta t_{i}^{-1}
$$

we will have the following results:

Theorem 2 (The $h_{2}$ control strategy): Consider the system described by (1) and controlled by (17), with $t_{i}$ defined as in (9), Under these assumptions, the following properties hold: $i$ ) $W_{N}=x_{T}$; and $\left.i i\right)\left\|x_{i}-W_{i}\right\| \leq \epsilon, i \in\{1, \ldots, N\}$. Assuming $\mathcal{U}$ defined as in (2), a sufficient condition for this theorem to hold is

$$
\left\|u_{0}\right\| \max _{i}\left(1+\frac{\gamma_{i}}{\left\|u_{0}\right\|}\right) \leq u_{\max }
$$

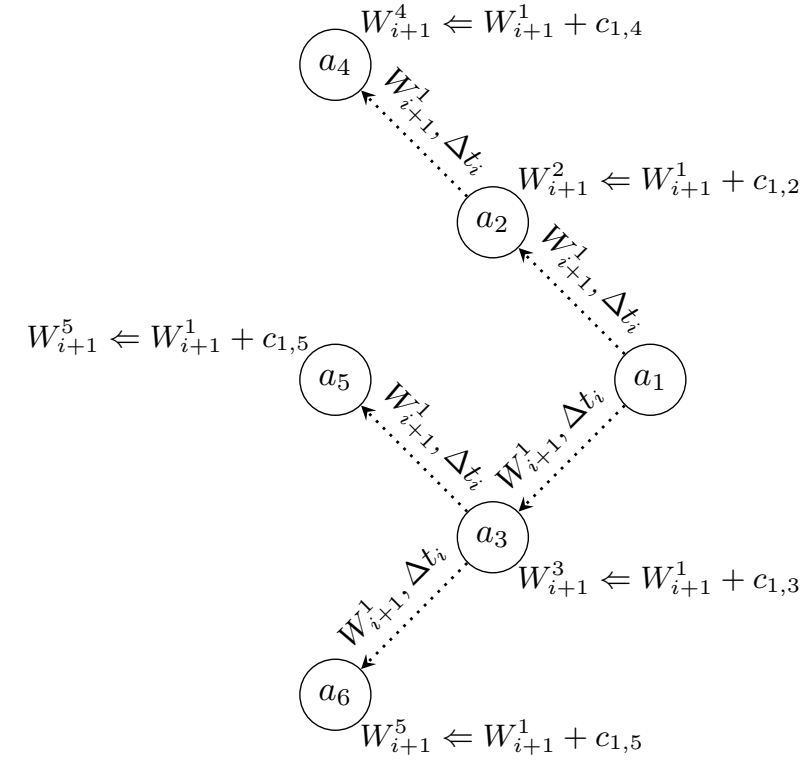

Fig. 1. Waypoint transmission procedure. This figure also represents the possibility of some agents acting as repeaters, due to the limited communication range.

Proof: The proof is omitted as it is a simple extension of the proof of theorem 2 in [11].

We have thus proposed two different control strategies that not only fulfill one of our initial requirements (both are able to reach the target in the specified time if there are no disturbances) but also, are able to position the agent within a distance $\epsilon$ of the target in the presence of disturbances.

\section{The Multi-Agent Case}

With the tools developed from the analysis of the single agent scenario we now turn to the original multi-agent problem. We use a similar procedure to what is shown in [11]. By developing a leader-follower approach, where one agent (the leader) is assigned the task of computing its next waypoint $W_{i}$ (exactly as in the single agent problem), but also to transmit it to the follower, together with the travel time $\Delta t_{i}$, we are able to gain some insight into the original problem.

Keeping the leader-follower structure, at time $t_{i}$ the leader computes $\Delta t_{i}$ and $W_{i+1}$, and transmits them to the rest of the network (illustrated in figure 1). Motivated by the formation property of the network, agent $j$ 's $i^{t h}$ waypoint is defined as:

$$
W_{i+1}^{j}=W_{i+1}^{L}+c_{L, j},
$$

where $L$ denotes the leader, $j$ denotes any other agent in the set $A$, and $c_{L, j}$ the desired relative position between the two agents. At time $t_{i}$, agent $j$ defines its control signal as:

$$
u_{i}^{j}=\left(W_{i+1}-x_{i}^{j}\right) \Delta t_{i}^{-1} .
$$

Having the leader use either one of the control strategies proposed in theorems 1 and 2, then the following properties hold, for all $j \in\{1, \ldots, M\}: i) W_{N}^{j}=x_{T}^{j}$; and 
ii) $\left\|x_{i}^{j}-W_{i}^{j}\right\| \leq \epsilon, \forall i \in\{1, \ldots, M\}$. This comes from the fact that, due to equation (19), the multi-agent network is, to some extent, equivalent to $M-1$ leader-follower networks, so the proofs developed for the two-agent network [11] can be applied, assuming conditions on the inter-agent distance hold. These are sufficient conditions imposed on $\epsilon$ in order do ensure that communication is preserved (the range condition)

$$
\epsilon \leq \min _{i \in A}\left[\frac{1}{2}\left(r-\max _{j \in \mathcal{N}(i)}\left(d_{i, j}\right)\right)\right]
$$

and that the agents do not collide with each other (collision avoidance condition)

$$
\epsilon<\frac{1}{2} d_{i^{*}, j^{*}}
$$

where $d_{i, j}=\left\|c_{i, j}\right\|$, and $i^{*}, j^{*}=\arg \min \left(d_{i, j}\right)_{i \neq j}$. These conditions were obtained using a conservative approach (hence the use of the word sufficient) and can thus lead to overly restrictive conditions on $\epsilon$.

\section{Disturbance SET ESTIMATION}

When developing our control strategy, we assumed that at every instant $t_{i}$ an upper bound on the disturbance, $\gamma_{i}$, would be available. We then proceeded by using this bound to determine the time it would take for the position uncertainty to reach the threshold value, $\epsilon$. It so happens that in many applications, the disturbance set is unknown and/or timevarying, so fulfilling our initial requirement may not be as simple.

In the multi-agent scenario, at every stopping instant each agent $j$ will be at a position $x_{i}^{j}$, very likely to be different from its target position, $W_{i}^{j}$. The difference between the two - the position drift $\delta_{i}^{j}$ - can be measured (as $W_{i}^{j}$ was computed at $t_{i-1}$ and $x_{i}^{j}$ directly measured at $t_{i}$ ) and used to determine the average disturbance speed for that agent during the interval $\left[t_{i-1}, t_{i}\right]$ :

$$
\bar{\omega}_{i}^{j}=\delta_{i}^{j}\left(t_{i}-t_{i-1}\right)^{-1}
$$

By taking advantage of the fact that we have a formation of $M$ agents, at every surfacing instant $t_{i}$ we will have $M$ different measurements of the average disturbance speed that we can use to characterize the disturbance set $\Omega$. One possible way to do this is by feeding the $k^{t h}$ available measurement to the following recursive estimators for the mean, $\mu$,

$$
\mu_{k}=\left(1-k^{-1}\right) \mu_{k-1}+k^{-1} \bar{\omega}_{k}
$$

and variance, $\Sigma$,

$$
\Sigma_{k}=\left(1-k^{-1}\right)\left[\Sigma_{k-1}+k^{-1}\left(\bar{\omega}_{k}-\mu_{k-1}\right)\left(\bar{\omega}_{k}-\mu_{k-1}\right)^{T}\right],
$$

Note that in order to consider the measurements of all agents for disturbance set estimation purposes, we must first assume that the set has a negligible spatial variation with respect to the formation - in other words, that the parameters describing the disturbance set are the same for the whole formation at every instant. These two parameters - the mean and variance - provide a way to determine the required bound on the disturbance speed. It should be mentioned that the way they are used to determine the bound will depend on the probability distribution function that is chosen to approximate the disturbance set. At the same time, by using estimates of the mean and variance we can no longer guarantee our positioning results, as underestimating these parameters, for example, may lead to a value of $\gamma_{i}$ that is not an upper bound on the disturbance set.

\section{A. A normally distributed disturbance set}

A likely candidate to approximate the disturbance set is the normal distribution, motivated by the fact that it can be used to describe ocean currents [13], as well as other relevant physical phenomena. However, before we make this consideration, we first need to introduce some relevant concepts.

The probability density function for a bivariate $\left(x \in \mathbb{R}^{2}\right)$ normal distribution is given by

$$
f_{x}(x)=\frac{1}{2 \pi \operatorname{det}(\Sigma)^{\frac{1}{2}}} e^{-\frac{1}{2}(x-\mu)^{T} \Sigma^{-1}(x-\mu)}
$$

where $\mu$ denotes the mean and $\Sigma$ the variance. In order to state some relevant results about this distribution, we will also need to introduce the following definition. An ellipsoid $\mathcal{E}$ with center $c \in \mathbb{R}^{n}$ and shape matrix $Q$, with $Q$ symmetric positive definite is defined by

$$
\mathcal{E}(c, Q)=\left\{x \in \mathbb{R}^{n}:(x-c)^{T} Q^{-1}(x-c) \leq 1\right\}
$$

The directions of the ellipsoid's axis are given by the eigenvectors of the matrix $Q$, with the lengths of the semi-axes given by the square root of the corresponding eigenvalue.

By considering a normally distributed disturbance set we are able to contemplate a greater number of external disturbances, and at the same time provide our system with some degree of increased robustness. This will, nevertheless, conflict with our uncertainty model, as the normal distribution is unbounded $\left(\Omega\right.$ will now be equal to $\mathbb{R}^{2}$ ) and, consequently, there will no longer be a value of $\gamma_{i}$ for which equation (7) holds. Still, we can use an extension of the empirical rule (also known as three-sigma rule) to the bivariate distribution. This rule allows us to determine the cumulative probability that the random variable lie in the ellipsoid $\mathcal{E}\left(\mu, k^{2} \Sigma\right)$ for different levels of $k$, as shown in Table I. These cumulative probability

\begin{tabular}{|c|c|}
\hline$k$ & $p\left(x \in \mathcal{E}\left(\mu, k^{2} \Sigma\right)\right)$ \\
\hline 1 & 0.3945 \\
\hline 2 & 0.8647 \\
\hline 3 & 0.9889 \\
\hline 4 & 0.9997 \\
\hline
\end{tabular}

TABLE I

CUMUlATIVE PROBABILITY ASSOCIATED WITH DIFFERENT ELLIPSOIDS.

values will thus influence the likelihood that our results on the agent's position hold. Consider, for example, that we have $\mu=[0,0]^{T}$ and $\Sigma=I$. This means that the probability of the 
average disturbance speed being less than or equal to $1 \mathrm{~m} / \mathrm{s}$ is of $0.3945,0.8647$ for $2 \mathrm{~m} / \mathrm{s}$ and so on. Assuming our mean and variance estimates are close to the true values, by setting $\gamma_{i}=2 \mathrm{~m} / \mathrm{s}$ we will have a probability of 0.8647 that equation (13) holds.

We have thus proposed a way to modify our strategy to handle unknown and/or time-varying disturbance sets, as well as the case of and unbounded, normally distributed set. Still, it should be stressed out that we were only able to do this by compromising the determinism of our initial results.

\section{NUMERICAL EXAMPLES}

In the following example we let our formation be such that $A=\left\{a^{1}, a^{2}, a^{3}, a^{4}, a^{5}\right\}, r=90, c_{1,2}=c_{2,4}=$ $[-50,50]^{T}, c_{1,3}=c_{3,5}=[-50,50]^{T}$. Additionally, we have that $x_{T}^{1}=[500,0]^{T}$ with $t_{T}=100$. The range and collision avoidance conditions on $\epsilon$ will lead us to $\epsilon \leq 9.65$ and $\epsilon \leq 25 \sqrt{2}$ respectively, so we set $\epsilon=10$. In this example we let the disturbance set be normally distributed, with $\Omega \sim\left([-1,1]^{T}, 0.5^{2} I\right)$, where $I$ denotes the 2 by 2 identity matrix. One way to define the upper bound on the disturbance speed is to let $\gamma=\left\|[-1,1]^{T}\right\|+2 \sigma \approx 2.5$. The formation trajectory results for this scenario are shown in figures 2 and 3, where the real and estimated trajectories are shown in red and blue respectively. The blue circles centered at the waypoints, with a radius of $\epsilon$ units represent the maximum position uncertainty. The true and estimated disturbance sets are shown in figure 4.

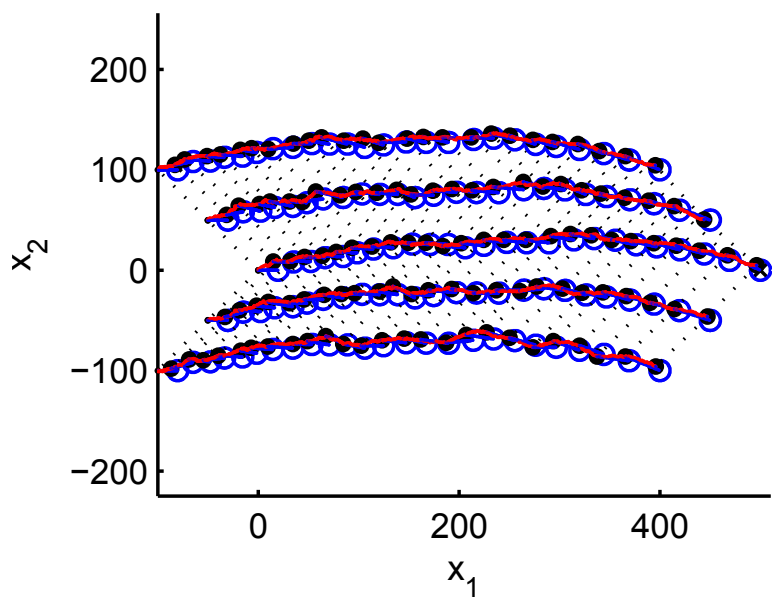

Fig. 2. Formation trajectory having the leader use the $h_{1}$ control strategy.

Alternatively, noticing that the mean disturbance is the same for all agent and, as such, will not affect formation in terms of collisions or range, we can set $\gamma=3 \sigma=1.5$. The formation trajectory results for this scenario are shown in figures 5 and 6 , and the true and estimated disturbance sets in figure 7 .

Looking at the trajectory plots for both choices of $\gamma$ we are able to see $h_{1}$ 's greater sensitivity (with respect to $h_{2}$ ) to the adverse component of the mean disturbance. This is evident if we look at the increasing distance between waypoints in figures 2 and 5, and also by noticing that all waypoints lie

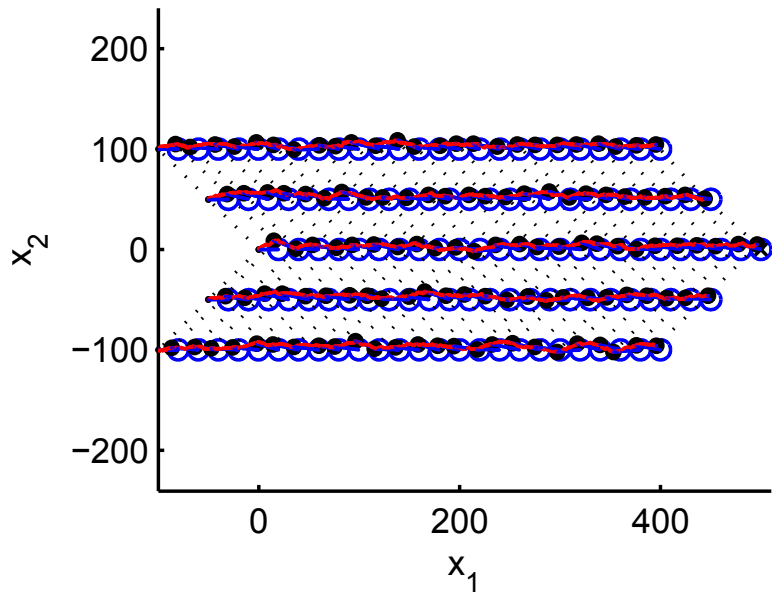

Fig. 3. Formation trajectory having the leader use the $h_{2}$ control strategy.

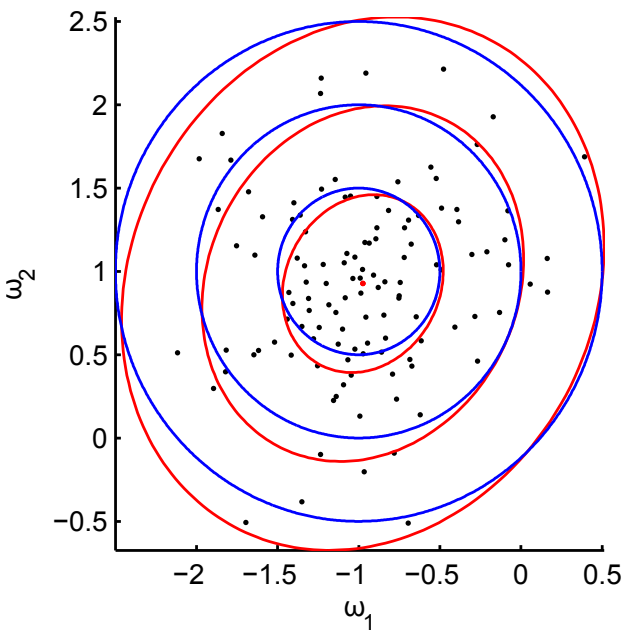

Fig. 4. Ellipsoids with shape matrices $\Sigma, 2^{2} \Sigma$ and $3^{2} \Sigma$ are plotted in blue and red for the true and estimated disturbance sets respectively. The black dots represent the average disturbance speed measurements.

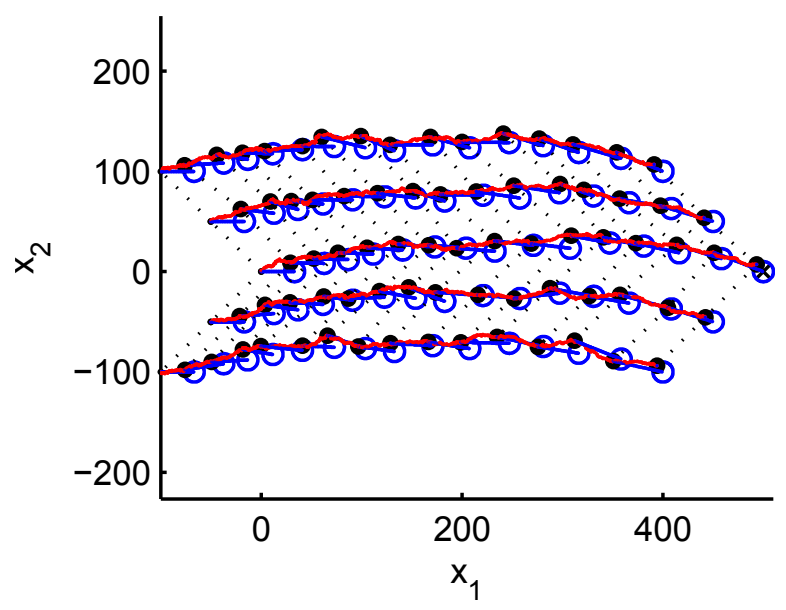

Fig. 5. Formation trajectory having the leader use the $h_{1}$ control strategy. 


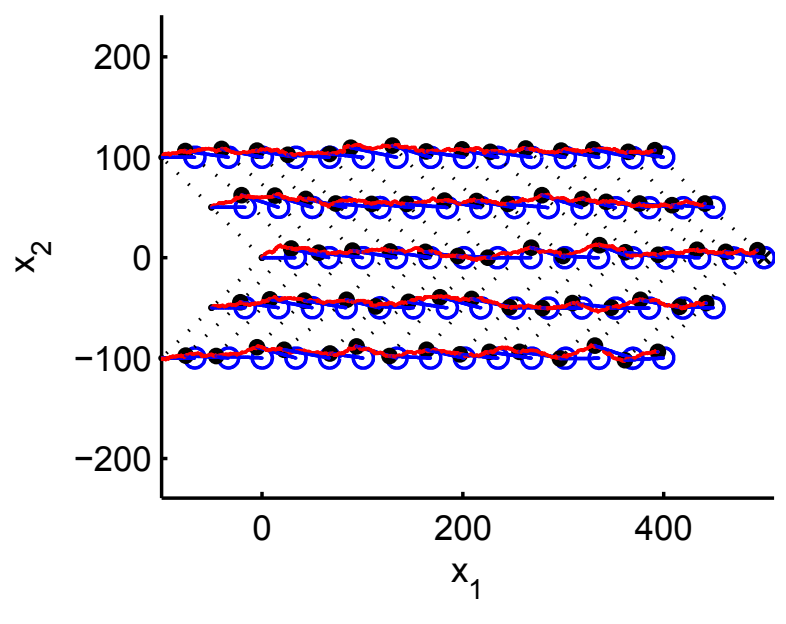

Fig. 6. Formation trajectory having the leader use the $h_{2}$ control strategy.

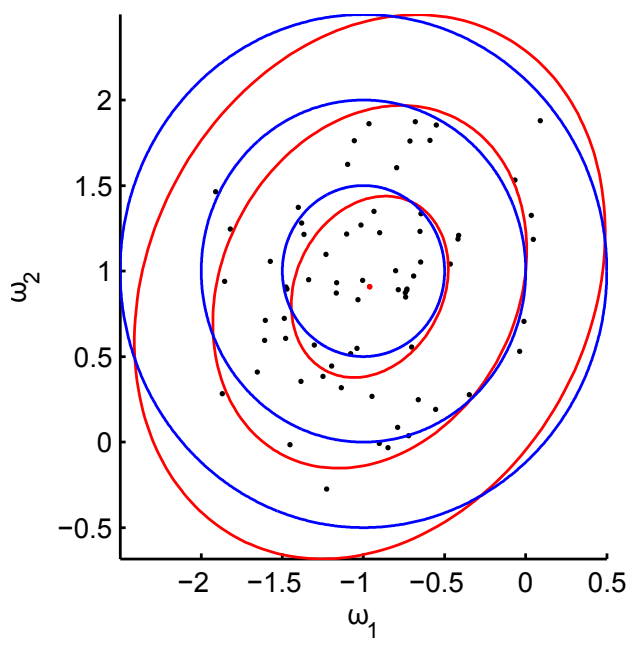

Fig. 7. Ellipsoids with shape matrices $\Sigma, 2^{2} \Sigma$ and $3^{2} \Sigma$ are plotted in blue and red for the true and estimated disturbance sets respectively. The black dots represent the average disturbance speed measurements.

along the ideal trajectory for $h_{2}$ (figures 3 and 6). Finally, looking at figures 4 and 7 we can see the impact of the reduction of the number of surfacing events in the disturbance set estimates.

\section{CONCLusions And Future Work}

We have proposed an event-based approach that relies on an uncertainty model to trigger surfacing events, so that the agents can measure their own position and, after receiving instructions from the leader, update their control signal. We also proposed a method to characterize the disturbance set using these events. This is an important step, as it is essential for the network to have an upper bound on the disturbances in order to compute the waypoints.

Future research directions include the development of a completely distributed approach, without the need for a leading agent. More complex, non-linear dynamics should also be contemplated. Finally, more explicit results on the implications of a normally distributed disturbance set should be derived, in order to achieve an increased degree of robustness to uncertainty.

\section{REFERENCES}

[1] J. P. Desai, J. Ostrowski, and V. Kumar, "Controlling formations of multiple mobile robots," Proc. of IEEE Int. Conf. on Robotics and Automation, pp. 2864-2869, 1998.

[2] Z. Lin, B. Francis, and M. Maggiore, "Necessary and sufficient graphical conditions for formation control of unicycles," IEEE Transactions on Automatic Control, vol. 50, no. 1, pp. 121-127, 2005.

[3] G. Lafferriere, A. Williams, J. Caughman, and J. Veerman, "Decentralized control of vehicle formations," Systems and Control Letters, vol. 54, no. 9, pp. 899-910, 2005.

[4] M. Haque and M. Egerstedt, "Decentralized formation selection mechanisms inspired by foraging bottlenose dolphins," Mathematical Theory of Networks and Systems, 2008.

[5] F. Xie and R. Fierro, "On motion coordination of multiple vehicles with nonholonomic constraints," American Control Conference, pp. 1888$1893,2007$.

[6] S. Mastellone, D. Stipanovic, C. Graunke, K. Intlekofer, and M. Spong, "Formation control and collision avoidance for multi-agent nonholonomic systems: Theory and experiments," International Journal of Robotics Research, vol. 27, no. 1, pp. 107-126, 2008.

[7] P. Ogren, E. Fiorelli, and N. Leonard, "Cooperative control of mobile sensor networks:adaptive gradient climbing in a distributed environment," Automatic Control, IEEE Transactions on, vol. 49, no. 8, pp. $1292-1302$, aug. 2004.

[8] N. Leonard and E. Fiorelli, "Virtual leaders, artificial potentials and coordinated control of groups," Decision and Control, 2001. Proceedings of the 40th IEEE Conference on, vol. 3, pp. 2968 -2973 vol.3, 2001.

[9] W. Yan, R. Cui, and D. Xu, "Formation control of underactuated autonomous underwater vehicles in horizontal plane," Automation and Logistics, 2008. ICAL 2008. IEEE International Conference on, pp. 822 -827, September 2008.

[10] J. Borges de Sousa, K. H. Johansson, J. Silva, and A. Speranzon, "A verified hierarchical control architecture for coordinated multi-vehicle operations," International Journal of Adaptive Control and Signal Processing, vol. 21, no. 2-3, pp. 159-188, 2007, special issue on autonomous adaptive control of vehicles.

[11] P. Teixeira, D. Dimarogonas, K. Johansson, and J. Sousa, "Multi-Agent Coordination with Event-based Communication," American Control Conference, 2010, to appear.

[12] E. Hernandez-Martinez and E. Aranda-Bricaire, "Non-collision conditions in multi-agent robots formation using local potential functions," Robotics and Automation, 2008. ICRA 2008. IEEE International Conference on, pp. 3776 -3781, May 2008.

[13] J. Mattias Green and A. Stigebrandt, "Statistical models and distributions of current velocities with application to the prediction of extreme events," Estuarine, Coastal and Shelf Science, vol. 58, no. 3, pp. $601-609,2003$. [Online]. Available: http://www.sciencedirect.com/science/article/B6WDV-49PYMF91/2/7ba2f54567a2c99f4fc0f94a5c072984 